\title{
UNIQUENESS OF SOLUTION OF A SINGULAR HEAT EQUATION
}

\author{
EUTIQUIO C. YOUNG \\ Department of Mathematics and Computer Science \\ Florida State University \\ Tallahassee, Florida 32306 \\ (Received May 20, 1983)
}

ABSTRACT. Some uniqueness results for the singular heat equation are presented in this paper.

KEY WORDS AND PHRASES. Singular Heat Equations, Uniqueness Theorem 1980 MATHEMATICS SUBJECT CLASSIFICATION CODE. 35K05

1. INTRODUCTINN.

In recent years several papers appeared on the uniqueness of solutions to il1posed problems for partial differential equations of various types. For example, in $[1],[2],[3],[4]$ conditions were obtained for the uniqueness of solutions of boundary value problems for hyperbolic equations. Corresponding results were obtained in [5], [6], [7] for ultrahyperbolic equations. In [8] the question was studied with an equation of mixed type, while in [9] and [10] it was extended to abstract differential equations. The arguments of [2] were modified in [11] to give an alternative proof of the known uniqueness theorem for the boundary value problem associated with the backward heat equation.

In this note we shall give some uniqueness results for the singular heat equation

$$
\mathrm{Lu}=\mathrm{u}_{t}-\sum_{i=1}^{\mathrm{n}}\left(\mathrm{u}_{\mathrm{x}_{i} \mathrm{x}_{i}}+\frac{\alpha_{i}}{\mathrm{x}_{i}} \mathrm{u}_{\mathrm{i}}\right)+\mathrm{c}(\mathrm{t}) \mathrm{u}=0
$$

where $\alpha_{i}$ is a real parameter, .. $\infty<\alpha_{i}<\infty, 1 \leq i \leq n$ and $c(t)$ is an integrable function on $0<t<T, T<\infty$. Adopting the method of [6], we first establish our results for a domain, $\mathrm{D}=\mathrm{H} \times(0, \mathrm{~T})$, where

$$
H=\left\{x: x=\left(x_{1}, \ldots, x_{n}\right), 0<x_{i}<a_{i} .1 \leq i \leq n\right\} .
$$


At the end, we shall make a remark concerning the validity of our theorems for an arbitrary domain $\mathrm{H}$ in $\mathrm{R}^{\mathrm{n}}$.

For convenience, we write

$$
x^{\alpha}=x_{1}^{\alpha}{ }_{1}^{1} \ldots x_{n}^{\alpha}, \quad \int_{H} f d x=\int_{0}^{a} f d x=\int_{0}^{a_{1}} \ldots \int_{0}^{a} f d x_{1} \ldots d x_{n}
$$

and adopt the convention that repeated indices are to be summed from 1 to $n$.

2. A LEMMA.

The following lemma is essential to the proof of our theorems.

Lemma. Let $-\infty<\alpha_{i}<\infty$ and $1 \leq i \leq n$. The eigenvalues and eigenfunctions of the problem

$$
\left(x^{\alpha} x_{x_{i}}\right)_{i}+x^{\alpha} \mu x=0 \text { in } H
$$

or

$$
\mathrm{X}(\mathrm{x})<\infty \quad \text { on } \mathrm{x}_{\mathrm{i}}=0 \text { if } \alpha_{i}>0
$$

and

$$
x(x)=0 \quad \text { on } x_{i}=0 \text { if } \alpha_{i} \leq 0
$$$$
\mathrm{x}(\mathrm{x})=0 \quad \text { on } \mathrm{x}_{i}=\mathrm{a}_{i}(1 \leq \mathrm{i} \leq \mathrm{n})
$$

are given by

$$
\mu=\mu_{1}+\cdot \cdot+\mu_{n}
$$

where $\mu_{i}$ is a root of

$$
\mathrm{J}_{\left|1-\alpha_{i}\right| / 2}\left(\sqrt{\mu}_{i} a_{i}\right)=0(1 \leq i \leq n)
$$

and

$$
\mathrm{X}(\mathrm{x})=\prod_{i=1}^{\mathrm{n}} \mathrm{x}_{i}\left(1-\alpha_{i}\right) / 2 \mathrm{~J}\left|1-\alpha_{i}\right| / 2\left(\sqrt{\mu}_{i} \mathrm{x}_{i}\right)
$$

If condition (2.3) is replaced by

$$
x_{x_{i}}(x)=0 \text { on } x_{i}=a_{i} \quad(1 \leq i \leq n),
$$

then the lemma holds provided $\mu_{i}$ satisfies

$$
\mathrm{J}_{\left|1-\alpha_{i}\right| / 2}\left(\sqrt{\mu}_{i} \mathrm{x}_{i}\right)=0 \quad(1 \leq \mathrm{i} \leq \mathrm{n}) \text {. }
$$

The lemma and the remark above can be established by the usual separation of variables argument together with the properties of Bessel functions. Further, we observe that in each case the set of eigenfunctions is complete with respect to the weight function $\mathrm{x}^{\alpha}$.

\section{MAIN RESULTS}

We now state and prove our uniqueness theorems.

THEOREM 1. Let $\alpha_{i}>0$ with $i=1, \ldots, n$. If $u \in C^{2}(D) \cap C^{1}(\bar{D})$ is a solution of the problem 


$$
\begin{aligned}
& \mathrm{Lu}=0 \text { in } D \\
& \mathrm{u}(\mathrm{x}, \mathrm{T})=0 \quad \mathrm{x} \in \mathrm{H} \\
& \mathrm{u}(\mathrm{x}, \mathrm{t})=0 \text { on } \mathrm{x}_{i}=\mathrm{a}_{i}(1 \leq i \leq \mathrm{n})
\end{aligned}
$$

for $0<t<T$, then $u=0$ identically in $D$.

PROOF. It is interesting to note that no boundary condition is prescribed for $\mathrm{u}$ on $\mathrm{x}_{i}=0$ with $\mathrm{i}=1, \ldots, \mathrm{n}$. To prove the theorem let $\mathrm{x}(\mathrm{x})$ be an eigenfunction of (2.1)-(2.3) for $\alpha_{i}>0$ corresponding to an eigenvalue $\mu$ as given in (2.4). Define

$$
v(t)=\int_{H} x^{\alpha} u(x, t) x(x) d x
$$

Since $\mathrm{Lu}=0$, we have, by $(2.1)$,

$$
\begin{aligned}
v^{\prime}(t) & =\int_{x^{\alpha} u_{t}(x, t) x(x) d x}^{H}\left[x^{\alpha}\left[\left(x^{\alpha} u_{x_{i}}\right) x_{i}-x^{\alpha} c u\right] x d x\right. \\
& =\int_{-}\left[x^{\alpha}\left(u_{x_{i}} x-u x_{x_{i}}\right)\right]_{x_{i}} d x \\
& -\int_{H}^{\alpha} x^{\alpha}(\mu+c) d x d x
\end{aligned}
$$

By the divergence theorem, the first integral on the right side of (3.3) becomes

$$
\int_{\partial H} x^{\alpha}\left(u_{x_{i}} x-u x_{x_{i}}\right) v_{i} d S
$$

where $\left(v_{1}, \ldots, v_{n}\right)$ denotes the outward unit normal vector on $\partial H$.

Since $\alpha_{i}>0$, the integral (3.4) vanishes on $x_{i}=0$ where $i=1, \ldots, n$. Likewise, it vanishes on $x_{i}=a_{i}(1 \leq i \leq n)$ since both $u$ and $x$ do. Hence (3.3) reduces to

$$
v^{\prime}(t)=-[\mu+c(t)] v(t)
$$

whose solution is given by

$$
v(t)=C \exp \left[-\left(\mu t+\int^{t} c(s) d s\right)\right] .
$$

Since $u(x, T)=0$, it follows from (3.2) that $v(T)=0$, so that (3.4) is identically zero, that is,

$$
\int_{H} x^{\alpha} u(x, t) x(x) d x=0 .
$$

This implies that $u$ is identically zero by the completeness of the set of eigenfunctions of (2.1)-(2.3).

Similarly, we have

THEOREM 2. Let $a_{i} \leq 0$ with $i=1, \ldots, n$. If $u \in C^{2}(D) \cap C^{1}(\bar{D})$ is a solution of the problem

$$
\begin{aligned}
& \mathrm{Lu}=0 \text { in } \mathrm{D} \\
& \mathrm{u}(\mathrm{x}, \mathrm{T})=0 \quad \mathrm{x} \in \mathrm{H} \\
& \mathrm{u}(\mathrm{x}, \mathrm{t})=0 \text { on } \mathrm{x}_{i}=0, \mathrm{a}_{i}(1 \leq \mathrm{i} \leq \mathrm{n}),
\end{aligned}
$$


then $\mathrm{u} \equiv 0$ in $\mathrm{D}$.

We need only note that in the proof the eigenfunction X of the problem (2.1)(2.3) for $\alpha_{i} \leq 0$ has the property that

$$
x^{\alpha} x(x)=0(x), \quad x^{\alpha} x_{x_{i}}(x)=0(1)
$$

so that the integral (3.4) vanishes, which again leads to (3.6).

4. Remarks.

It is readily seen that by using the eigenfunctions of the problem (2.1), (2.2), (2.7), Theorems 1 and 2 remain valid when the boundary condition on $x_{i}=a_{i}$ $(1 \leq i \leq n)$ is replaced by $u_{x_{i}}=0$. Also, Theorem 1 holds for an arbitrary bounded domain $\mathrm{H}$ in $\mathrm{R}_{+}^{\mathrm{n}}$ with a piecewise smooth boundary which includes a portion of the coordinate planes $x_{i}=0, i=1, \ldots, n$. The condition on $x_{i}=a_{i}$ with $i=1, \ldots, n$ now has to be prescribed on $\partial \mathrm{H}$ where $\mathrm{x}_{i} \neq 0$. For the proof, we assume that the associated eigenvalue problem has a complete set of eigenfunctions. However, since we need to know the behavior of the eigenfunctions on $x_{i}=0$ when $\alpha_{i} \leq 0, i=1, \ldots, n$, our proof of Theorem 2 fails for an arbitrary domain $\mathrm{H}$.

\section{REFERENCES}

1. ABDUL-LATIF, A.I. and DIAZ, J.B., Dirichlet, Neumann, and mixed boundary value problems for the wave equation $u_{x x}-u_{y y}=0$ for a rectangle, Applicable Anal. 1 (1971), 1-12.

2. DUNNINGER, D.R. and ZACHMANOGLOU,E.C., The condition for uniqueness of the Dirichlet problem for hyperbolic equations in cylindrical domains. J. Math. Mech. 18 (1969), $763-766$.

3. TRAVIS, C.C. On the uniqueness of solutions to hyperbolic boundary value problems, Trans. Amer. Math. Soc. 216(1976), 327-336.

4. YOUNG, E.C., Uniqueness of solutions of the Dirichlet and Neumann problems for hyperbolic equations, Trans. Amer. Math. Soc. 160(1971), 403-409.

5. DIAZ, J.B. and YOUNG, E.C., Uniqueness of solutions of certain boundary value problems for ultrahyperbolic equations, Amer. Math. Soc. 29(1971), 569-574.

6. TRAVIS, C.C. and YOUNG, E.C., Uniqueness of solutions to singular boundary value problems, SIAM, J.Math. Ana1. 8 (1977), 111-117.

7. YOUNG, E.C., Uniqueness of solutions of the Dirichlet problem for singular ultrahyperbolic equations, Proc. Amer. Math. Soc. 36 (1972), 130-136.

8. NAKHUSHEV, A.M., A ciriterion for the uniqueness of the solution of Dirichlet problem for an equation of mixed type in a cylindrical domain, J. Diff. Eqs. $\underline{6}(1970), 150-151$.

9. DUNNINGER, D.R. and LEVINE, H.A., Uniqueness criteria for solutions to singular boundary value problems, Trans. Amer. Math. Soc. 221(1976), 289-301.

10. DUNNINGER,D.R. and LEVINE, H.A., Uniqueness criteria for solutions to abstract boundary value problems, J. Diff. Eqs. 22(1976), 368-378.

11. Sigillito, V.G., On the uniqueness of solutions of certain improperly posed problems, Proc. Amer. Math. Soc. 24(1970), 828-831. 


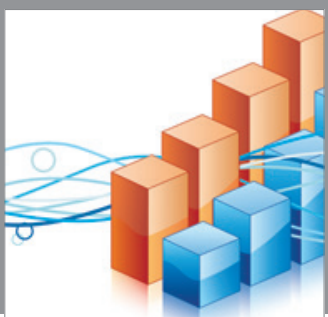

Advances in

Operations Research

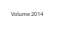

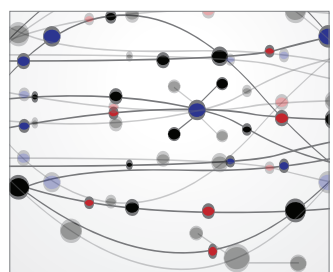

\section{The Scientific} World Journal
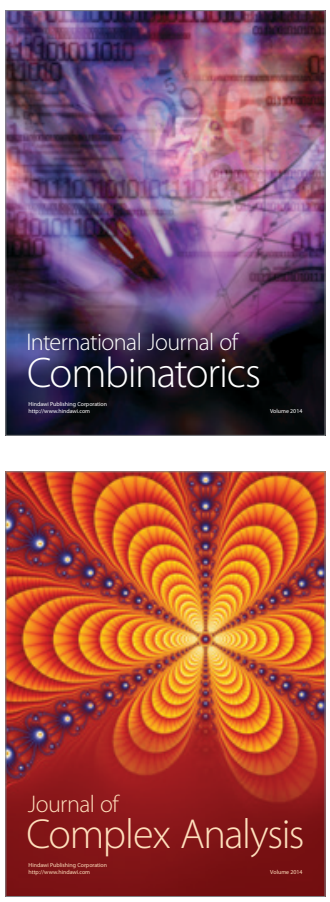

International Journal of

Mathematics and

Mathematical

Sciences
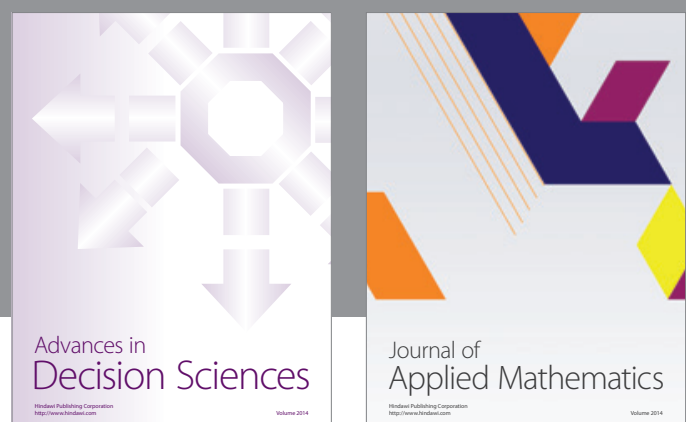

Journal of

Applied Mathematics
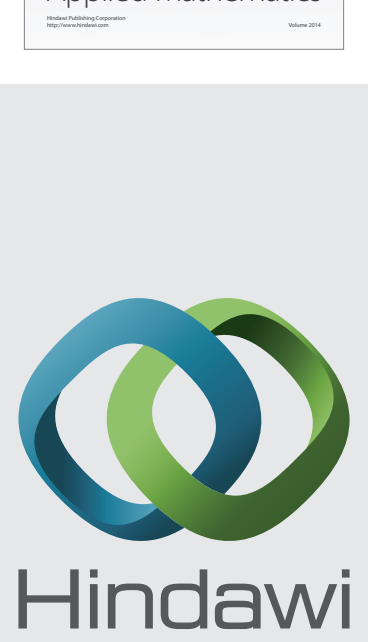

Submit your manuscripts at http://www.hindawi.com
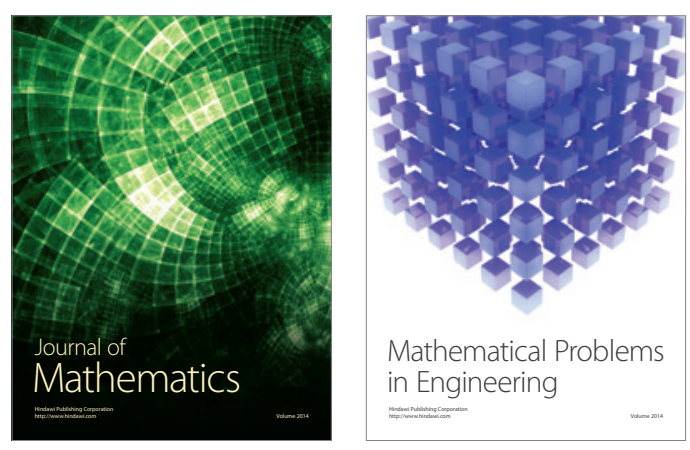

Mathematical Problems in Engineering
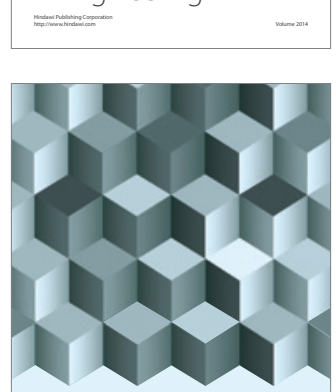

Journal of

Function Spaces
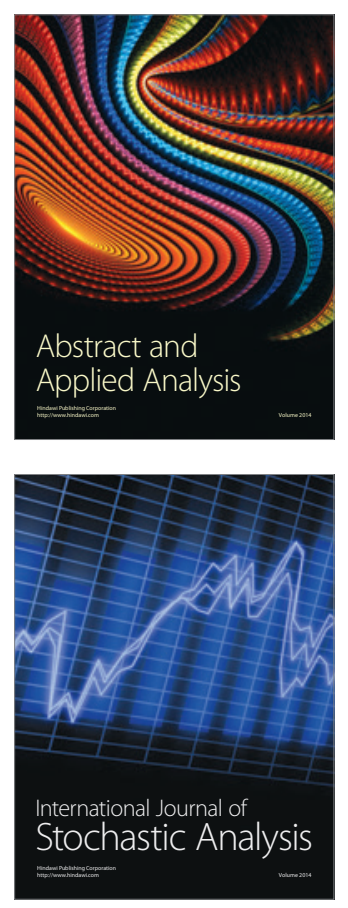

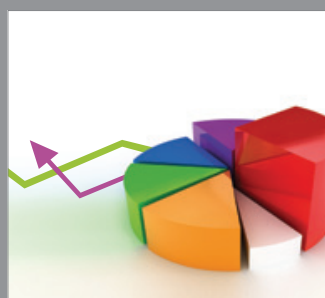

ournal of

Probability and Statistics

Promensencen
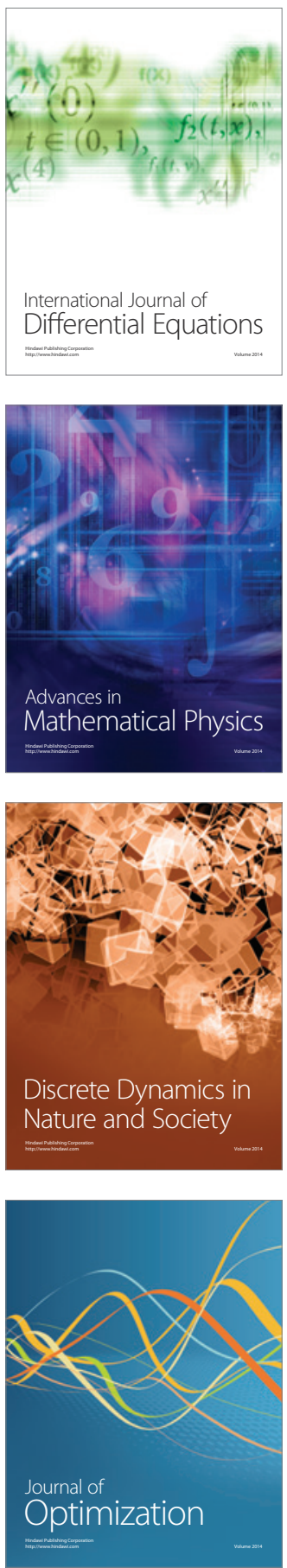